International Journal of Current Advanced Research

ISSN: O: 2319-6475, ISSN: P: 2319 - 6505, Impact Factor: SJIF: 5.995

Available Online at www.journalijcar.org

Volume 6; Issue 3; March 2017; Page No. 2351-2357

DOI: http://dx.doi.org/10.24327/ijcar.2017.2357.0008

\title{
ON THE LOCATION OF ZEROS OF POLYNOMIALS
}

\section{Gulzar M.H}

Department of Mathematics, University of Kashmir, Srinagar

\section{A R T I C L E I N F O}

\section{Article History:}

Received $9^{\text {th }}$ December, 2016

Received in revised form $14^{\text {th }}$ January, 2017

Accepted $12^{\text {th }}$ February, 2017

Published online $28^{\text {th }}$ March, 2017

\section{A B S T R A C T}

In this paper we restrict the real and imaginary parts of the coefficients of a polynomial and find a region containing all its zeros. In addition to being generalizations of some known results, our results give many other interesting results for particular choices of the parameters.

\section{Key words:}

Coefficients, Polynomial, Zeros.

Copyright $\bigcirc 2017$ Dhanalakshmi, B. This is an open access article distributed under the Creative Commons Attribution License, which permits unrestricted use, distribution, and reproduction in any medium, provided the original work is properly cited.

\section{INTRODUCTION}

As for the region containing all the zeros of a polynomial with real monotonically decreasing positive coefficients, Enestrom and kakeya proved the following elegant result known as the Enestrom-Kakeya Theorem $[3,4]$ :

Theorem A: all the zeros of a polynomial $P(z)=\sum_{j=0}^{n} a_{j} z^{j}$ satisfying $a_{n} \geq a_{n-1} \geq \ldots \ldots \geq a_{1} \geq a_{0}>0$ lie in $|z| \leq 1$.

Various generalizations and extensions of this result are available in the literature. Recently Gulzar [2] proved the following result:

Theorem A: Let $P(z)=\sum_{j=0}^{n} a_{j} z^{j}$ be a polynomial of degree $n$ with $\operatorname{Re}\left(a_{j}\right)=\alpha_{j}, \operatorname{Im}\left(a_{j}\right)=\beta_{j}, j=0,1,2, \ldots \ldots, n$ such that for some $\lambda, \mu, 0 \leq \lambda \leq n-1,0 \leq \mu \leq n-1$

and for some $k_{1}, k_{2} \geq 1$,

$k_{1}{ }^{n-\lambda+1} \alpha_{n} \geq k_{1}{ }^{n-\lambda} \alpha_{n-1} \geq \ldots \ldots \geq k_{1}^{2} \alpha_{\lambda+1} \geq k_{1} \alpha_{\lambda}$,

$k_{2}{ }^{n-\mu+1} \beta_{n} \geq k_{2}{ }^{n-\mu} \beta_{n-1} \geq \ldots . . \geq k_{2}{ }^{2} \beta_{\mu} \geq k_{2} \beta_{\mu}$,

and

$L=\left|\alpha_{\lambda}-\alpha_{\lambda-1}\right|+\left|\alpha_{\lambda-1}-\alpha_{\lambda-2}\right|+\ldots \ldots+\left|\alpha_{1}-\alpha_{0}\right|+\left|\alpha_{0}\right|$,

$M=\left|\beta_{\mu}-\beta_{\mu-1}\right|+\left|\beta_{\mu-1}-\beta_{\mu-2}\right|+\ldots \ldots+\left|\beta_{1}-\beta_{0}\right|+\left|\beta_{0}\right|$. 
Then all the zeros of $\mathrm{P}(\mathrm{z})$ lie in

$$
\begin{aligned}
& \left|z+\frac{\left(k_{1}-1\right) \alpha_{n}+i\left(k_{2}-1\right) \beta_{n}}{a_{n}}\right| \leq \frac{1}{\left|a_{n}\right|}\left[\alpha_{n}+\beta_{n}+\left(k_{1}-1\right) \sum_{j=\lambda+1}^{n}\left(\left|\alpha_{j}\right|+\alpha_{j}\right)-\left(k_{2}-1\right) \sum\left(\left|\beta_{j}\right|+\beta_{j}\right)\right. \\
& \left.-\left(k_{1}-1\right)\left|\alpha_{n}\right|-\left(k_{2}-1\right)\left|\beta_{n}\right|+L+M-\alpha_{\lambda}-\beta_{\mu}\right] .
\end{aligned}
$$

\section{MAIN RESULTS}

In this paper we prove the following result:

Theorem 1: Let $P(z)=\sum_{j=0}^{n} a_{j} z^{j}$ be a polynomial of degree $n$ with $\operatorname{Re}\left(a_{j}\right)=\alpha_{j}, \operatorname{Im}\left(a_{j}\right)=\beta_{j}, j=0,1,2, \ldots \ldots, n$ such that for some $\lambda, \mu, 0 \leq \lambda \leq n-1,0 \leq \mu \leq n-1$

and for some $k_{1}, k_{2} \leq 1$,

$k_{1}{ }^{n-\lambda+1} \alpha_{n} \leq k_{1}{ }^{n-\lambda} \alpha_{n-1} \leq \ldots \ldots \leq k_{1}{ }^{2} \alpha_{\lambda+1} \leq k_{1} \alpha_{\lambda}$,

$k_{2}{ }^{n-\mu+1} \beta_{n} \leq k_{2}{ }^{n-\mu} \beta_{n-1} \leq \ldots . . \leq k_{2}{ }^{2} \beta_{\mu} \leq k_{2} \beta_{\mu}$,

and

$L=\left|\alpha_{\lambda}-\alpha_{\lambda-1}\right|+\left|\alpha_{\lambda-1}-\alpha_{\lambda-2}\right|+\ldots \ldots+\left|\alpha_{1}-\alpha_{0}\right|+\left|\alpha_{0}\right|$,

$M=\left|\beta_{\mu}-\beta_{\mu-1}\right|+\left|\beta_{\mu-1}-\beta_{\mu-2}\right|+\ldots \ldots+\left|\beta_{1}-\beta_{0}\right|+\left|\beta_{0}\right|$.

Then all the zeros of $\mathrm{P}(\mathrm{z})$ lie in

$\left|z-\frac{\left(1-k_{1}\right) \alpha_{n}+i\left(1-k_{2}\right) \beta_{n}}{a_{n}}\right| \leq \frac{1}{\left|a_{n}\right|}\left[\alpha_{\lambda}+\beta_{\mu}-\left(k_{1} \alpha_{n}+k_{2} \beta_{n}\right)+L+M\right.$

$\left.+\left(1-k_{1}\right) \sum_{j=\lambda+1}^{n-1}\left(\alpha_{j}+\left|\alpha_{j}\right|\right)+\left(1-k_{2}\right) \sum_{j=\mu+1}^{n-1}\left(\beta_{j}+\left|\beta_{j}\right|\right)\right]$

Further the number of zeros of $\mathrm{P}(\mathrm{z})$ in $\frac{\left|a_{0}\right|}{X} \leq|z| \leq \frac{R}{c}, c>1, R \geq 1$ does not exceed $\frac{1}{\log c} \log \frac{X+\left|a_{0}\right|}{\left|a_{0}\right|}=\frac{1}{\log c} \log \left(1+\frac{X}{\left|a_{0}\right|}\right)$ and the number of zeros of $\mathrm{P}(\mathrm{z})$ in $\frac{\left|a_{0}\right|}{Y} \leq|z| \leq \frac{R}{c}, c>1, R \leq 1$ does not exceed $\frac{1}{\log c} \log \frac{Y+\left|a_{0}\right|}{\left|a_{0}\right|}=\frac{1}{\log c} \log \left(1+\frac{Y}{\left|a_{0}\right|}\right)$, where $\mathrm{R}$ is any positive number and

$$
\begin{aligned}
& X=\left|a_{n}\right| R^{n+1}+R^{n}\left[\alpha_{\lambda}+\beta_{\mu}-\left(\alpha_{n}+\beta_{n}\right)+\left(1-k_{1}\right) \sum_{j=\lambda+1}^{n}\left(\left|\alpha_{j}\right|+\alpha_{j}\right)\right. \\
& \left.+\left(1-k_{2}\right) \sum_{j=\mu+1}^{n}\left(\left|\beta_{j}\right|+\beta_{j}\right)\right], R \geq 1 \\
& Y=\left|a_{n}\right| R^{n+1}+R\left[\alpha_{\lambda}+\beta_{\mu}-\left(\alpha_{n}+\beta_{n}\right)+\left(1-k_{1}\right) \sum_{j=\lambda+1}^{n}\left(\left|\alpha_{j}\right|+\alpha_{j}\right)\right. \\
& \left.+\left(1-k_{2}\right) \sum_{j=\mu+1}^{n}\left(\left|\beta_{j}\right|+\beta_{j}\right)\right], R \leq 1 .
\end{aligned}
$$

For different values of the parameters in Theorem 1, we get different interesting results. For example, for $k_{1}=k_{2}=1$, Theorem 1 gives the following result:

Corollary 1: Let $P(z)=\sum_{j=0}^{n} a_{j} z^{j}$ be a polynomial of degree $n$ with $\operatorname{Re}\left(a_{j}\right)=\alpha_{j}, \operatorname{Im}\left(a_{j}\right)=\beta_{j}, j=0,1,2, \ldots \ldots, n$ such that for some $\lambda, \mu, 0 \leq \lambda \leq n-1,0 \leq \mu \leq n-1$, 
$\alpha_{n} \leq \alpha_{n-1} \leq \ldots \ldots \leq \alpha_{\lambda+1} \leq \alpha_{\lambda}$

$\beta_{n} \leq \beta_{n-1} \leq \ldots . . \leq \beta_{\mu} \leq \beta_{\mu}$,

and

$L=\left|\alpha_{\lambda}-\alpha_{\lambda-1}\right|+\left|\alpha_{\lambda-1}-\alpha_{\lambda-2}\right|+\ldots \ldots+\left|\alpha_{1}-\alpha_{0}\right|+\left|\alpha_{0}\right|$,

$M=\left|\beta_{\mu}-\beta_{\mu-1}\right|+\left|\beta_{\mu-1}-\beta_{\mu-2}\right|+\ldots \ldots+\left|\beta_{1}-\beta_{0}\right|+\left|\beta_{0}\right|$.

Then all the zeros of $\mathrm{P}(\mathrm{z})$ lie in

$|z| \leq \frac{1}{\left|a_{n}\right|}\left[\alpha_{\lambda}+\beta_{\mu}-\left(\alpha_{n}+\beta_{n}\right)+L+M\right]$.

Further the number of zeros of $\mathrm{P}(\mathrm{z})$ in $\frac{\left|a_{0}\right|}{X} \leq|z| \leq \frac{R}{c}, c>1, R \geq 1$ does not exceed $\frac{1}{\log c} \log \frac{X+\left|a_{0}\right|}{\left|a_{0}\right|}=\frac{1}{\log c} \log \left(1+\frac{X}{\left|a_{0}\right|}\right)$ and the number of zeros of $\mathrm{P}(\mathrm{z})$ in $\frac{\left|a_{0}\right|}{Y} \leq|z| \leq \frac{R}{c}, c>1, R \leq 1$ does not exceed $\frac{1}{\log c} \log \frac{Y+\left|a_{0}\right|}{\left|a_{0}\right|}=\frac{1}{\log c} \log \left(1+\frac{Y}{\left|a_{0}\right|}\right)$, where $\mathrm{R}$ is any positive number and

$$
\begin{array}{ll}
X=\left|a_{n}\right| R^{n+1}+R^{n}\left[\alpha_{\lambda}+\beta_{\mu}-\left(\alpha_{n}+\beta_{n}\right)+L+M\right] & , R \geq 1 \\
Y=\left|a_{n}\right| R^{n+1}+R\left[\alpha_{\lambda}+\beta_{\mu}-\left(\alpha_{n}+\beta_{n}\right)+L+M\right] & , R \leq 1 .
\end{array}
$$

Taking $\lambda=\mu=0$ in Theorem 1, we get the following result:

Corollary 2: Let $P(z)=\sum_{j=0}^{n} a_{j} z^{j}$ be a polynomial of degree $n$ with $\operatorname{Re}\left(a_{j}\right)=\alpha_{j}, \operatorname{Im}\left(a_{j}\right)=\beta_{j}, j=0,1,2, \ldots \ldots, n$ such that for some $0<k_{1}, k_{2} \leq 1$,

$k_{1}^{n} \alpha_{n} \leq k_{1}^{n-1} \alpha_{n-1} \leq \ldots \ldots \leq k_{1} \alpha_{1} \leq \alpha_{0}$,

$k_{2}{ }^{n} \beta_{n} \leq k_{2}{ }^{n-1} \beta_{n-1} \leq \ldots . . . \leq k_{2} \beta_{\mu} \leq \beta_{0}$.

Then all the zeros of $\mathrm{P}(\mathrm{z})$ lie in

$\left|z-\frac{\left(1-k_{1}\right) \alpha_{n}+i\left(1-k_{2}\right) \beta_{n}}{a_{n}}\right| \leq \frac{1}{\left|a_{n}\right|}\left[\alpha_{0}+\beta_{0}-\left(k_{1} \alpha_{n}+k_{2} \beta_{n}\right)+\left|\alpha_{0}\right|+\left|\beta_{0}\right|\right]$.

Further the number of zeros of $\mathrm{P}(\mathrm{z})$ in $\frac{\left|a_{0}\right|}{X} \leq|z| \leq \frac{R}{c}, c>1, R \geq 1$ does not exceed $\frac{1}{\log c} \log \frac{X+\left|a_{0}\right|}{\left|a_{0}\right|}=\frac{1}{\log c} \log \left(1+\frac{X}{\left|a_{0}\right|}\right)$ and the number of zeros of $\mathrm{P}(\mathrm{z})$ in $\frac{\left|a_{0}\right|}{Y} \leq|z| \leq \frac{R}{c}, c>1, R \leq 1$ does not exceed $\frac{1}{\log c} \log \frac{Y+\left|a_{0}\right|}{\left|a_{0}\right|}=\frac{1}{\log c} \log \left(1+\frac{Y}{\left|a_{0}\right|}\right)$, where $\mathrm{R}$ is any positive number and

$$
\begin{array}{ll}
X=\left|a_{n}\right| R^{n+1}+R^{n}\left[\alpha_{0}+\beta_{0}-\left(\alpha_{n}+\beta_{n}\right)+\left|\alpha_{0}\right|+\left|\beta_{0}\right|\right] & , R \geq 1 \\
Y=\left|a_{n}\right| R^{n+1}+R\left[\alpha_{0}+\beta_{0}-\left(\alpha_{n}+\beta_{n}\right)+\left|\alpha_{0}\right|+\left|\beta_{0}\right|\right. & , R \leq 1 .
\end{array}
$$

\section{Lemmas}

For the proofs of the above result, we need the following lemmas:

Lemma 1: Let $\mathrm{f}(\mathrm{z})$ (not identically zero) be analytic for $|z| \leq R, f(0) \neq 0$ and $f\left(a_{k}\right)=0, k=1,2, \ldots \ldots, n$. Then 
$\frac{1}{2 \pi} \int_{0}^{2 \pi} \log \mid f\left(\operatorname{Re}^{i \theta}|d \theta-\log | f(0) \mid=\sum_{j=1}^{n} \log \frac{R}{\left|a_{j}\right|}\right.$.

Lemma 2 is the famous Jensen's Theorem (see page 208 of [1]).

Lemma 2: Let $\mathrm{f}$ (z) be analytic for $|z| \leq R, f(0) \neq 0$ and $|f(z)| \leq M$ for $|z| \leq R$. Then the number of zeros of $\mathrm{f}(\mathrm{z})$ in $|z| \leq \frac{R}{c}, c>1$ does not exceed $\frac{1}{\log c} \log \frac{M}{|f(0)|}$.

Lemma 2 is a simple deduction from Lemma 1.

\section{Proof of Theorem 1}

Consider the polynomial

$$
\begin{aligned}
& F(z)=(1-z) P(z) \\
&=(1-z)\left(a_{n} z^{n}+a_{n-1} z^{n-1}+\ldots \ldots+a_{1} z+a_{0}\right) \\
&=-a_{n} z^{n+1}+\left(a_{n}-a_{n-1}\right) z^{n}+\ldots \ldots+\left(a_{\lambda+1}-a_{\lambda}\right) z^{\lambda+1}+\left(a_{\lambda}-a_{\lambda-1}\right) z^{\lambda} \\
&+\ldots \ldots .\left(a_{1}-a_{0}\right) z+a_{0} \\
&=-a_{n} z^{n+1}-\left(k_{1}-1\right) \alpha_{n} z^{n}+\left(k_{1} \alpha_{n}-\alpha_{n-1}\right) z^{n}+\left(k_{1} \alpha_{n-1}-\alpha_{n-2}\right) z^{n-1}+\ldots \ldots \\
& \quad+\left(k_{1} \alpha_{\lambda+1}-\alpha_{\lambda}\right) z^{\lambda+1}+\left(\alpha_{\lambda}-\alpha_{\lambda-1}\right) z^{\lambda}+\ldots \ldots .+\left(\alpha_{1}-\alpha_{0}\right) z+\alpha_{0} \\
&-\left(k_{1}-1\right)\left(\alpha_{n-1} z^{n-1}+\alpha_{n-2} z^{n-2}+\ldots \ldots .+\alpha_{\lambda+1} z^{\lambda+1}\right) \\
&+ i\left\{\left(k_{2} \beta_{n}-\beta_{n-1}\right) z^{n}-\left(k_{2}-1\right) \beta_{n} z^{n}+\left(k_{2} \beta_{n-1}-\beta_{n-2}\right) z^{n-1}+\ldots \ldots\right. \\
&+\left(k_{2} \beta_{\mu+1}-\beta_{\mu}\right) z^{\mu+1}-\left(k_{2}-1\right)\left(\beta_{n-1} z^{n-1}+\ldots \ldots .+\beta_{\mu+1} z^{\mu+1}\right)+\left(\beta_{\mu}-\beta_{\mu-1}\right) z^{\mu} \\
&+\left.\ldots \ldots . .+\left(\beta_{1}-\beta_{0}\right) z+\beta_{0}\right\}
\end{aligned}
$$

For $|z|>1$ so that $\frac{1}{|z|^{j}}<1, \forall j=1,2, \ldots \ldots, n$, we have, by using the hypothesis

$|F(z)| \geq\left|a_{n} z+\left(k_{1}-1\right) \alpha_{n}+i\left(k_{2}-1\right) \beta_{n} \| z\right|^{n}-\left[\left|k_{1} \alpha_{n}-\alpha_{n-1}\left\|\left.z\right|^{n}+\left|k_{1} \alpha_{n-1}-\alpha_{n-2} \| z\right|^{n-1}+\ldots \ldots\right.\right.\right.$

$+\left|k_{1} \alpha_{\lambda+1}-\alpha_{\lambda}\left\|\left.z\right|^{\lambda+1}+\left|\alpha_{\lambda}-\alpha_{\lambda-1}\right||z|^{\lambda}+\ldots . .+\left|\alpha_{1}-\alpha_{0} \| z\right|+\left|\alpha_{0}\right|\right.\right.$

$+\left(1-k_{1}\right)\left(\left|\alpha_{n-1}\right||z|^{n-1}+\ldots . .+\left|\alpha_{\lambda+1}\right||z|^{\lambda+1}\right)+\left|k_{2} \beta_{n}-\beta_{n-1} \| z\right|^{n}+\left|k_{2} \beta_{n-1}-\beta_{n-2}\right||z|^{n-1}$

$+\ldots . .+\left|k_{2} \beta_{\mu+1}-\beta_{\mu}\right||z|^{\mu+1}+\left|\beta_{\mu}-\beta_{\mu-1}\right||z|^{\mu}+\ldots \ldots+\left|\beta_{1}-\beta_{0}\right||z|+\left|\beta_{0}\right|$

$\left.+\left(1-k_{2}\right)\left(\left|\beta_{n-1} \| z\right|^{n-1}+\ldots . .+\left|\beta_{\mu+1}\right||z|^{\mu+1}\right)\right]$

$=|z|^{n}\left[\left|a_{n} z+\left(k_{1}-1\right) \alpha_{n}+i\left(k_{2}-1\right) \beta_{n}\right|-\left[\left|k_{1} \alpha_{n}-\alpha_{n-1}\right|+\frac{\left|k_{1} \alpha_{n-1}-\alpha_{n-2}\right|}{|z|}+\frac{\left|k_{1} \alpha_{n-2}-\alpha_{n-3}\right|}{|z|^{2}}\right.\right.$

$+\ldots . .+\frac{\left|k_{1} \alpha_{\lambda+1}-\alpha_{\lambda}\right|}{|z|^{n-\lambda-1}}+\frac{\left|\alpha_{\lambda}-\alpha_{\lambda-1}\right|}{|z|^{n-\lambda}}+\ldots . .+\frac{\left|\alpha_{1}-\alpha_{0}\right|}{|z|^{n-1}}+\frac{\left|\alpha_{0}\right|}{|z|^{n}}+\left(k_{1}-1\right)\left(\frac{\left|\alpha_{n-1}\right|}{|z|}+\ldots \ldots\right.$.

$\left.+\frac{\left|\alpha_{\lambda+1}\right|}{|z|^{n-\lambda-1}}\right)+\left|k_{2} \beta_{n}-\beta_{n-1}\right|+\frac{\left|k_{2} \beta_{n-1}-\beta_{n-2}\right|}{|z|}+\ldots . .+\frac{\left|k_{2} \beta_{\mu+1}-\beta_{\mu}\right|}{|z|^{n-\mu-1}}+\frac{\left|\beta_{\mu}-\beta_{\mu-1}\right|}{|z|^{n-\mu}}$

$\left.+\ldots . .+\frac{\left|\beta_{1}-\beta_{0}\right|}{|z|^{n-1}}+\frac{\left|\beta_{0}\right|}{|z|^{n}}+\left(k_{2}-1\right)\left(\frac{\left|\beta_{n-1}\right|}{|z|}+\ldots . .+\frac{\left|\beta_{\mu+1}\right|}{|z|^{n-\mu-1}}\right)\right]$

$>|z|^{n}\left[\left|a_{n} z+\left(k_{1}-1\right) \alpha_{n}+i\left(k_{2}-1\right) \beta_{n}\right|-\left\{\left|k_{1} \alpha_{n}-\alpha_{n-1}\right|+\left|k_{1} \alpha_{n-1}-\alpha_{n-2}\right|+\left|k_{1} \alpha_{n-2}-\alpha_{n-3}\right|\right.\right.$ 
$+\ldots \ldots+\left|k_{1} \alpha_{\lambda+1}-\alpha_{\lambda}\right|+\left|\alpha_{\lambda}-\alpha_{\lambda-1}\right|+\ldots \ldots+\left|\alpha_{1}-\alpha_{0}\right|+\left|\alpha_{0}\right|+\left(1-k_{1}\right)\left(\left|\alpha_{n-1}\right|+\ldots \ldots\right.$

$\left.+\left|\alpha_{\lambda+1}\right|\right)+\left|k_{2} \beta_{n}-\beta_{n-1}\right|+\left|k_{2} \beta_{n-1}-\beta_{n-2}\right|+\ldots \ldots+\left|k_{2} \beta_{\mu+1}-\beta_{\mu}\right|+\left|\beta_{\mu}-\beta_{\mu-1}\right|$

$\left.\left.+\ldots \ldots+\left|\beta_{1}-\beta_{0}\right|+\left|\beta_{0}\right|+\left(1-k_{2}\right)\left(\left|\beta_{n-1}\right|+\ldots . .+\left|\beta_{\mu+1}\right|\right)\right\}\right]$

$=|z|^{n}\left[\left|a_{n} z+\left(k_{1}-1\right) \alpha_{n}+i\left(k_{2}-1\right) \beta_{n}\right|-\left\{\alpha_{n-1}-k_{1} \alpha_{n}+\alpha_{n-2}-k_{1} \alpha_{n-1}+\alpha_{n-3}-k_{1} \alpha_{n-2}\right.\right.$

$+\ldots \ldots+\alpha_{\lambda}-k_{1} \alpha_{\lambda+1}+L+\left(1-k_{1}\right)\left(\left|\alpha_{n-1}\right|+\ldots \ldots+\left|\alpha_{\lambda+1}\right|\right)+\beta_{n-1}-k_{2} \beta_{n}$

$\left.\left.+\beta_{n-2}-k_{2} \beta_{n-1}+\ldots . .+\beta_{\mu}-k_{2} \beta_{\mu+1}+M+\left(1-k_{2}\right)\left(\left|\beta_{n-1}\right|+\ldots \ldots+\left|\beta_{\mu+1}\right|\right)\right\}\right]$

$=|z|^{n}\left[\left|a_{n} z-\left\{\left(1-k_{1}\right) \alpha_{n}+i\left(1-k_{2}\right) \beta_{n}\right\}\right|-\left\{\alpha_{\lambda}+\beta_{\mu}-\left(k_{1} \alpha_{n}+k_{2} \beta_{n}\right)+L+M\right.\right.$

$\left.\left.+\left(1-k_{1}\right) \sum_{j=\lambda+1}^{n-1}\left(\alpha_{j}+\left|\alpha_{j}\right|\right)+\left(1-k_{2}\right) \sum_{j=\mu+1}^{n-1}\left(\beta_{j}+\left|\beta_{j}\right|\right)\right\}\right]$

$>0$

if

$\left|a_{n} z-\left\{\left(1-k_{1}\right) \alpha_{n}+i\left(1-k_{2}\right) \beta_{n}\right\}\right|>\alpha_{\lambda}+\beta_{\mu}-\left(k_{1} \alpha_{n}+k_{2} \beta_{n}\right)+L+M$

$+\left(1-k_{1}\right) \sum_{j=\lambda+1}^{n-1}\left(\alpha_{j}+\alpha_{j} \mid\right)+\left(1-k_{2}\right) \sum_{j=\mu+1}^{n-1}\left(\beta_{j}+\left|\beta_{j}\right|\right)$

i.e. if

$\left|z-\frac{\left(1-k_{1}\right) \alpha_{n}+i\left(1-k_{2}\right) \beta_{n}}{a_{n}}\right|>\frac{1}{\left|a_{n}\right|}\left[\alpha_{\lambda}+\beta_{\mu}-\left(k_{1} \alpha_{n}+k_{2} \beta_{n}\right)+L+M\right.$

$\left.+\left(1-k_{1}\right) \sum_{j=\lambda+1}^{n-1}\left(\alpha_{j}+\alpha_{j} \mid\right)+\left(1-k_{2}\right) \sum_{j=\mu+1}^{n-1}\left(\beta_{j}+\left|\beta_{j}\right|\right)\right]$.

This shows that those zeros of $F(z)$ whose modulus is greater than 1 lie in

$\left|z-\frac{\left(1-k_{1}\right) \alpha_{n}+i\left(1-k_{2}\right) \beta_{n}}{a_{n}}\right| \leq \frac{1}{\left|a_{n}\right|}\left[\alpha_{\lambda}+\beta_{\mu}-\left(k_{1} \alpha_{n}+k_{2} \beta_{n}\right)+L+M\right.$

$\left.+\left(1-k_{1}\right) \sum_{j=\lambda+1}^{n-1}\left(\alpha_{j}+\alpha_{j} \mid\right)+\left(1-k_{2}\right) \sum_{j=\mu+1}^{n-1}\left(\beta_{j}+\left|\beta_{j}\right|\right)\right]$.

Since the zeros of $\mathrm{F}(\mathrm{z})$ whose modulus is less than or equal to 1 already satisfy the above inequality and since the zeros of $\mathrm{P}(\mathrm{z})$ are also the zeros of $\mathrm{F}(\mathrm{z})$, it follows that all the zeros of $\mathrm{P}(\mathrm{z})$ lie in

$\left|z-\frac{\left(1-k_{1}\right) \alpha_{n}+i\left(1-k_{2}\right) \beta_{n}}{a_{n}}\right| \leq \frac{1}{\left|a_{n}\right|}\left[\alpha_{\lambda}+\beta_{\mu}-\left(k_{1} \alpha_{n}+k_{2} \beta_{n}\right)+L+M\right.$

$\left.+\left(1-k_{1}\right) \sum_{j=\lambda+1}^{n-1}\left(\alpha_{j}+\alpha_{j} \mid\right)+\left(1-k_{2}\right) \sum_{j=\mu+1}^{n-1}\left(\beta_{j}+\left|\beta_{j}\right|\right)\right]$.

Again

$F(z)=a_{0}+G(z)$

where

$$
\begin{aligned}
G(z)=-a_{n} z^{n+1} & -\left(k_{1}-1\right) \alpha_{n} z^{n}+\left(k_{1} \alpha_{n}-\alpha_{n-1}\right) z^{n}+\left(k_{1} \alpha_{n-1}-\alpha_{n-2}\right) z^{n-1}+\ldots \ldots \\
& +\left(k_{1} \alpha_{\lambda+1}-\alpha_{\lambda}\right) z^{\lambda+1}+\left(\alpha_{\lambda}-\alpha_{\lambda-1}\right) z^{\lambda}+\ldots \ldots+\left(\alpha_{1}-\alpha_{0}\right) z \\
& -\left(k_{1}-1\right)\left(\alpha_{n-1} z^{n-1}+\alpha_{n-2} z^{n-2}+\ldots \ldots . \alpha_{\lambda+1} z^{\lambda+1}\right) \\
& +i\left\{\left(k_{2} \beta_{n}-\beta_{n-1}\right) z^{n}-\left(k_{2}-1\right) \beta_{n} z^{n}+\left(k_{2} \beta_{n-1}-\beta_{n-2}\right) z^{n-1}+\ldots \ldots\right. \\
& +\left(k_{2} \beta_{\mu+1}-\beta_{\mu}\right) z^{\mu+1}-\left(k_{2}-1\right)\left(\beta_{n-1} z^{n-1}+\ldots \ldots+\beta_{\mu+1} z^{\mu+1}\right)+\left(\beta_{\mu}-\beta_{\mu-1}\right) z^{\mu} \\
& \left.+\ldots \ldots+\left(\beta_{1}-\beta_{0}\right) z\right\} .
\end{aligned}
$$


For $|z|=R, R>0$, we have

$$
\begin{aligned}
& |G(z)| \leq\left|a_{n}\right| R^{n+1}+\left(1-k_{1}\right)\left|\alpha_{n}\right| R^{n}+\left(\alpha_{n-1}-k_{1} \alpha_{n}\right) R^{n}+\left(\alpha_{n-2}-k_{1} \alpha_{n-1}\right) R^{n-1}+\ldots \ldots \\
& +\left(\alpha_{\lambda}-k_{1} \alpha_{\lambda+1}\right) R^{\lambda+1}+\left|\alpha_{\lambda}-\alpha_{\lambda-1}\right| R^{\lambda}+\ldots \ldots+\left|\alpha_{1}-\alpha_{0}\right| R \\
& +\left(1-k_{1}\right)\left(\left|\alpha_{n-1}\right| R^{n-1}+\left|\alpha_{n-2}\right| R^{n-2}+\ldots \ldots .+\alpha_{\lambda+1} \mid R^{\lambda+1}\right) \\
& +\left(\beta_{n-1}-k_{2} \beta_{n}\right) R^{n}+\left(1-k_{2}\right)\left|\beta_{n}\right| R^{n}+\left(\beta_{n-2}-k_{2} \beta_{n-1}\right) R^{n-1}+\ldots \ldots \\
& +\left(\beta_{\mu}-k_{2} \beta_{\mu+1}\right) R^{\mu+1}+\left(1-k_{2}\right)\left(\left|\beta_{n-1}\right| R^{n-1}+\ldots \ldots+\left|\beta_{\mu+1}\right| R^{\mu+1}\right)+\left|\beta_{\mu}-\beta_{\mu-1}\right| R^{\mu} \\
& \left.+\ldots \ldots+\left|\beta_{1}-\beta_{0}\right| R\right\} \\
& \leq\left|a_{n}\right| R^{n+1}+R^{n}\left[\left(1-k_{1}\right)\left|\alpha_{n}\right|+\alpha_{n-1}-k_{1} \alpha_{n}+\alpha_{n-2}-k_{1} \alpha_{n-1}+\ldots \ldots+\alpha_{\lambda}-k_{1} \alpha_{\lambda+1}+L\right. \\
& +\left(1-k_{1}\right)\left(\left|\alpha_{n-1}\right|+\ldots \ldots+\left|\alpha_{\lambda+1}\right|\right)+\beta_{n-1}-k_{2} \beta_{n} \\
& \left.+\beta_{n-2}-k_{2} \beta_{n-1}+\ldots \ldots+\beta_{\mu}-k_{2} \beta_{\mu+1}+M+\left(1-k_{2}\right)\left(\left|\beta_{n-1}\right|+\ldots \ldots+\left|\beta_{\mu+1}\right|\right)\right] \\
& =\left|a_{n}\right| R^{n+1}+R^{n}\left[\alpha_{\lambda}+\beta_{\mu}-\left(\alpha_{n}+\beta_{n}\right)+\left(1-k_{1}\right) \sum_{j=\lambda+1}^{n}\left(\left|\alpha_{j}\right|+\alpha_{j}\right)\right. \\
& \left.+\left(1-k_{2}\right) \sum_{j=\mu+1}^{n}\left(\left|\beta_{j}\right|+\beta_{j}\right)\right] \\
& =X
\end{aligned}
$$

for $R \geq 1$ and

for $R \leq 1$

$$
\begin{gathered}
|G(z)| \leq\left|a_{n}\right| R^{n+1}+R\left[\alpha_{\lambda}+\beta_{\mu}-\left(\alpha_{n}+\beta_{n}\right)+\left(1-k_{1}\right) \sum_{j=\lambda+1}^{n}\left(\left|\alpha_{j}\right|+\alpha_{j}\right)\right. \\
\left.+\left(1-k_{2}\right) \sum_{j=\mu+1}^{n}\left(\left|\beta_{j}\right|+\beta_{j}\right)\right] \\
=Y
\end{gathered}
$$

Since $\mathrm{G}(0)=0$ and $\mathrm{G}(\mathrm{z})$ is analytic for $|z| \leq R$, it follows, by Schwarz Lemma, that for $|z| \leq R$,

$|G(z)| \leq X|z|$ for $R \geq 1$ and $|G(z)| \leq Y|z|$ for $R \leq 1$.

Hence, for $|z| \leq R, R \geq 1$

if

$$
\begin{aligned}
|F(z)| & =\left|a_{0}+G(z)\right| \\
& \geq\left|a_{0}\right|-|G(z)| \\
& \geq\left|a_{0}\right|-X|z| \\
& >0
\end{aligned}
$$

$$
|z|<\frac{\left|a_{0}\right|}{X}
$$

Similarly, for $|z| \leq R, R \leq 1,|F(z)|>0$ if $|z|<\frac{\left|a_{0}\right|}{Y}$. 
In other words, $\mathrm{F}(\mathrm{z})$ does not vanish in $|z|<\frac{\left|a_{0}\right|}{X}$ for $R \geq 1$ and $\mathrm{F}(\mathrm{z})$ does not vanish in $|z|<\frac{\left|a_{0}\right|}{Y}$ for $R \leq 1$ in $|z| \leq R$. That means all the zeros of $\mathrm{F}(\mathrm{z})$ and hence all the zeros of $\mathrm{P}(\mathrm{z})$ lie in $|z| \geq \frac{\left|a_{0}\right|}{X}$ for $R \geq 1$ and in $|z| \geq \frac{\left|a_{0}\right|}{Y}$ that for $R \leq 1$ in $|z| \leq R$

Since for $|z| \leq R,|F(z)| \leq X+\left|a_{0}\right|$ for $R \geq 1$ and $|F(z)| \leq Y+\left|a_{0}\right|$ for $R \leq 1$ and since

$F(0)=a_{0} \neq 0$, it follows by Lemma 2 that the number of zeros of $\mathrm{P}(\mathrm{z})$ in $\frac{\left|a_{0}\right|}{X} \leq|z| \leq \frac{R}{c}, c>1, R \geq 1$ does not exceed $\frac{1}{\log c} \log \frac{X+\left|a_{0}\right|}{\left|a_{0}\right|}=\frac{1}{\log c} \log \left(1+\frac{X}{\left|a_{0}\right|}\right)$ and the number of zeros of $\mathrm{P}(\mathrm{z})$ in $\frac{\left|a_{0}\right|}{Y} \leq|z| \leq \frac{R}{c}, c>1, R \leq 1$ does not exceed $\frac{1}{\log c} \log \frac{Y+\left|a_{0}\right|}{\left|a_{0}\right|}=\frac{1}{\log c} \log \left(1+\frac{Y}{\left|a_{0}\right|}\right)$.

That proves Theorem 1 completely.

\section{References}

1. L. V. Ahlfors, Complex Analysis, $3^{\text {rd }}$ edition, Mc-Grawhill.

2. M.H.Gulzar, B.A.Zargar, A.W.Manzoor, Location of Zeros of Polynomials, International Journal of Computational Engineering Research,Vol.7, Issue 3, March 2017, 9-15.

3. M. Marden, Geometry of Polynomials, Math. Surveys No. 3, Amer. Math.Soc.(1966).

4. Q. I. Rahman and G. Schmeisser, Analytic Theory of Polynomials, Oxford University Press, New York (2002).

\section{Please cite this article in press as:}

Gulzar M.H (2017), On the Location of Zeros of Polynomials, International Journal of Current Advanced Research, 6(3), pp. 2351-2357

http://dx.doi.org/10.24327/ijcar.2017. 2357.0008 